\title{
Streptococcus pneumoniae Antigen Measurement
}

National Cancer Institute

\section{Source}

National Cancer Institute. Streptococcus pneumoniae Antigen Measurement. NCI

Thesaurus. Code C122152.

The determination of the amount of Streptococcus pneumoniae antigen present in a sample. 